\title{
On the implied volatility extraction and the selection of suitable kernel
}

\author{
Miloš Kopa, Sebastiano Vitali, Tomáš Tichý \\ Department of Econometrics \\ Institute of Information Theory and Automation of the \\ ASCR \\ Prague, Czech Republic
}

\author{
Tomáš Tichý \\ Department of Finance \\ VSB-TU Ostrava \\ Ostrava, Czech Republic \\ tomas.tichy@vsb.cz
}

\begin{abstract}
At the market, we can identify various kinds of options. Some of them are traded at organized exchanges and are quite liquid. Others are traded only between particular parties. The current market practice is to obtain implied volatility of liquid options as based on Black-Scholes type (BS hereafter) models. Such volatility is subsequently used to price illiquid or even exotic options. It therefore follows that the BS model at one time moment can be related to the whole set of IVs as given by maturity/moneyness relation of tradable options. One can therefore get IV curve or surface (a so called smirk or smile). Since the moneyness and maturity of IV often do not match the data of valuated options, some sort of estimating and local smoothing is necessary. However, it can lead to arbitrage opportunity, if no-arbitrage conditions on state price density (SPD) are ignored. In this paper, using option data on DAX index, we aim on the analyses of the behavior of IV and SPD with respect to different choices of bandwidth parameter $h$ and identification of a set of bandwidths which violates no-arbitrage conditions. We document that the change of $h$ implies interesting changes in the violation interval of moneyness. Finally, we also show the impact of $h$ on the total area of SPD under zero, which can be seen as a degree of no-arbitrage violation.
\end{abstract}

Keywords - arbitrage opportunity, implied volatility, option pricing, time grid, state price density

\section{INTRODUCTION}

At the market, we can identify various kinds of options. Some of them are traded at organized exchanges and are quite liquid. Others are traded only between particular parties. The volume of traded options increased sharply in 70's just after introducing the famous Black and Scholes model [3]. Thus, at that time the practice was to assume Gaussian distribution as a reliable proxy to the empirical observations of stock price or FX rate returns. Soon however, it was documented that the returns can be very far from the assumption of Gaussianity and thus the Black and Scholes model can be used only indirectly - take the market price of liquid option, invert the Black and Scholes formula, obtain a volatility (ie. implied volatility), put it into the formula by setting the parameters of illiquid option and get the price.

For example, [6] or [1] show for S\&P 500 options (ie one of the most liquid underlying assets) that implied volatility is not flat but can be strongly curved with changing maturity or/and moneyness. We call the behavior of the implied volatility its curve (assuming just one variable, ie. moneyness) or surface (assuming both the maturity and moneyness). Clearly, the behavior can be very different for various markets and underlying assets, which is probably related to particular market imperfections, such as restricted borrowing or lending. Such differences are evident especially when FX rate options are compared with equity options. Obviously, the set of parameters is not continuous and therefore, some nonparametric smoothing (and extrapolation) is needed to estimate the implied volatility function.

When we extract the implied volatility curve or surface from market prices of liquid options, we can use them to price the illiquid options or even options exotic, which we can trade only OTC. These, however, mostly have different parameters (moneyness, maturity) than those of traded options.

Notwithstanding, the implied volatility function must be calculated carefully - there exist several conditions on the price of call and put options, that must be fulfilled. Otherwise an arbitrage opportunity can arise, i.e., riskless profit higher than common riskless return. Clearly, there exist many technics that can be used to adjust the observations and transform them into smooth function.

In this paper, and in line with [2], we apply relatively classic approach of local polynomial smoothing techniques and study the bandwidth selection process in more details of recent data of DAX option prices (December 2011). In particular, we change $h$ and examine the impact on the interval of moneyness that brings arbitrage opportunity and on the total degree of noarbitrage violation.

We proceed as follows. In the following section we briefly review the problem of option pricing [7]. Next, we provide some basic facts about the implied volatility modeling and analyze the behavior of the implied volatility surface and potential arbitrage error for a given day using DAX options data. 


\section{OPTION VALUATION AND THE CONCEPT OF IMPLIED VOLATILITY}

Options are nonlinear types of financial derivatives, which gives the holder the right (but not the obligation) to buy the underlying asset in the future (at maturity time) at prespecified exercise price. Simultaneously, the writer of the option has to deliver the underlying asset if the holder asks.

Options can be classified due to a whole range of criterions, such as counterparty position (short and long), maturity time, complexity of the payoff function, etc. The basic features are the underlying asset $(S)$, which should be specified as precisely as possible (it is important mainly for commodities), the exercise price (K), and the maturity time $(T)$

If the option can be exercised only at maturity time $\mathrm{T}$, we call it the European option. By contrast, if it can be exercised also at any time prior the maturity day, ie. $t \in[0, T]$, we refer to it as the American option. A special type of options, possible to be classified somewhere between European and American options is the Bermudan option, which can be exercised at final number of times during the option life.

In dependency on the complexity of the payoff function, we usually distinguish simple plain vanilla options (PV) and exotic options. However, by a plain vanilla option we generally mean call and put options with the most simple payoff function. Sometimes, by plain vanilla options we mean any option which is regularly traded at the market, ie. it is liquid and no special formula is needed to obtain its price.

Thus,

$$
\begin{aligned}
& \qquad \Psi_{\text {call }}^{\text {vanilla }}=\left(S_{T}-K\right)^{+} \\
& \text {for vanilla call, and } \Psi_{\text {put }}^{\text {vanilla }}=\left(K-S_{T}\right)^{+}
\end{aligned}
$$

for vanilla put, where $(x)^{+} \equiv \max (x, 0)$.

Due to the definition of an option - it gives a right, but not an obligation to make a particular trade - we can deduce basic differences between the short and the long position. While the payoff resulting from the long position is non-negative, either 0 or $S_{T}-K$, the payoff of the short position will never be positive, ie. it is either $K-S_{T}$ or 0 . Moreover, it is obvious, that the long call payoff is not limited from above, but the short position payoff function goes only up to the exercise price (underlying asset price is zero).

Options are quite important type of financial derivatives since they allow to fit even very specific fears (hedging) and outlooks (speculation) about the future evolution. Due to the nonlinear payoff function and potential high sensitivity to changes in the input factors, such as volatility or even maturity, options are very challenging also for modeling purposes.

Obviously, since the standard option valuation model of Black and Scholes (and Merton) was based on the assumption of normally distributed returns, the presence of skewness and kurtosis at the market complicates the situation significantly. A common market practice is to use the market price as an exogenous variable to be put into the BS formula [3]. Thus, a so called implied volatility is obtained, ie. a number that assures that BS model provides the right price. Such implied volatility can subsequently be used to value even exotic options, which are not traded at the market.

Generally, the price of European option $f$ at time $t$ with maturity $T$ and payoff function $\Psi$ is given by the payoff expected under risk neutral probabilities $Q$ discounted by the risk less rate to the beginning $(t)$, ie. by setting $\square=T-t$ :

$$
f_{t}=e^{-r \tau} E_{t, T}^{Q}\left[\Psi_{T}\right]
$$

since the payoff at maturity is obviously identical to the European option value at the same time.

For example, assuming the payoff function of plain vanilla call and the normal distribution we get the valuation formula as follows (BS model for vanilla call):

$$
f_{\text {call }}^{\text {vanilla }}\left(\tau, S, K, r_{,} \sigma\right)=S F_{N}\left(d_{+}\right)-e^{-r \tau} K F_{N}\left(d_{-}\right)
$$

Here, $S$ is the underlying asset price at the valuation time $(t)$ and it is supposed to follow log-normal distribution, $\square$ is the time to maturity, $r$ is riskless rate valid over $\square, \square$ is the volatility expected over the same period, both per annum, and $F_{N}(x)$ is distribution function for standard normal distribution.

If the price of some options is available from the market, we can invert the formula to obtain the implied volatility, ie. the number that makes the formula equal to market price. Besides the important works, whose authors analyzed the impact of implied volatility on option price, belongs, besides others Dupire [4], who formulated a process followed by the underlying asset price in dependency on the moneyness and maturity, and Rubinstein [6], who formulated a discrete time model, the implied binomial tree.

Obviously, the implied volatility will differ for various input data, especially due to the moneyness (relation of the spot price and exercise price) and the time to maturity otherwise the model could not provide correct price. The dependency of the implied volatility on these two factors can be explained by the risk of jumps in the underlying asset price or other deviations from the assumption of Gaussianity. For example, Yan [8] carefully analyzed the impact of jump risk on the slope of the implied volatility function, which is informally referred to as the smile, and showed some interesting relations between the returns and the slope.

Although there exist many various approaches for the construction of the volatility curve or surface, including some recent alternatives, such as the application of radial basis function (see eg. [5] and references therein), we follow here relatively conservative approach adopted by [2]

\section{ANALYSIS CONCERNING THE THREE DIMENSIONS CASE}

In this section we present the analysis concerning the three dimensions case. We use as dataset all the options on DAX listed on 30 December 2011 with all the maturities. Following [2] we compute the unconstrained estimation of the IV surface. In Figure 1 we show the estimation with Epanechnikov kernel function, for moneyness bandwidth $h_{\kappa}=0.04$ and for maturity (calendar) bandwidth $h_{\tau}=1$. The historical data (black dots) are well described by the estimated surface. The IV smile is clear for small maturities and becomes less noticeable as the maturity increases. 


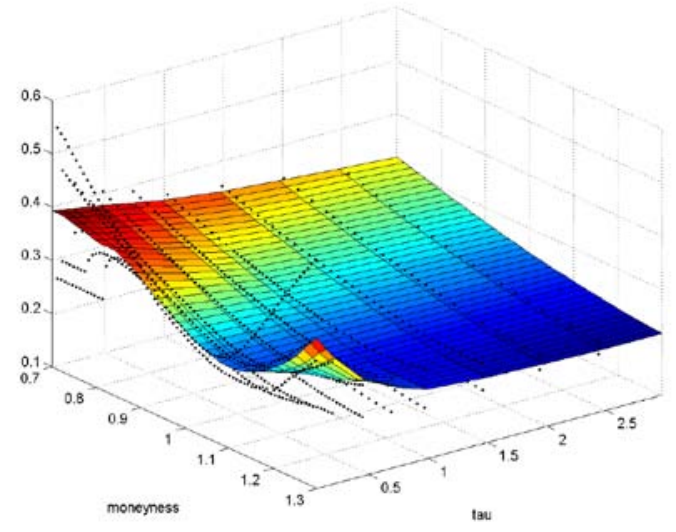

Figure 1. 3D estimation of Implied volatility of DAX options

In order to analyze the arbitrage presence, we produce the corresponding estimations for the state price density (SPD) again using fixed calendar bandwidth $h_{\tau}=1$.

The computation is done again with Epanechnikov kernel function and with three representative bandwidths for moneyness $h_{\kappa}=0.03,0.04,0.06$ and bandwidth $h_{\tau}=1$ for maturity. Besides each surface we propose also cuts for maturities equal to 168 days and 266 days. The negative parts of SPD violates the moneyness arbitrage free condition, see [2] for more details. Hence, the results show some arbitrage behavior for moneyness around 0.8 . We study more in deep the case with $h_{x}=0.04$ (see Figure 2).

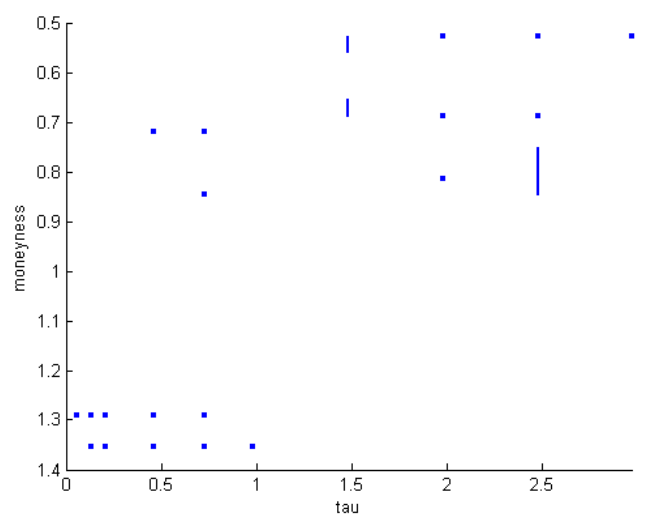

Figure 2. Arbitrage intervals for various maturities

In Figure 2 we show the arbitrage moneyness intervals (intervals with negative SPD) for each maturity. It is clear that the arbitrage chance is real and does not depend on the maturity. Indeed, the critical situations seem to be persistent among the increasing maturities. To evaluate the size of the arbitrage we compute Arbitrage measure as the volume of negative SPD for all considered moneyness bandwidths $(0.03$ $-0.08)$. The larger the measure is, the stronger arbitrage free violation is presented.

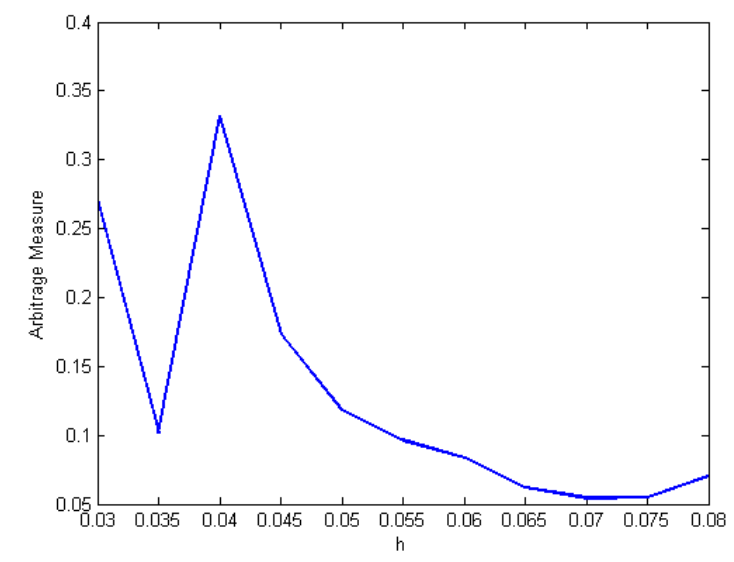

Figure 3. Arbitrage measure for DAX options IV surface

From Figure 3 we can notice that the curve is not strictly decreasing and this reinforce the idea that the magnitude of the arbitrage is not depending by the choice of the bandwidth but it is a genuine feature of the market. Moreover, to investigate the Arbitrage measure for various types of the kernel functions, we compare the Arbitrage measure for the different kernel functions and for three bandwidths used previously in Table 1.

Table 1. Arbitrage measure volume for DAX options IV surface

\begin{tabular}{|l|l|l|l|}
\hline & 0.03 & 0.04 & 0.06 \\
\hline Uniform & 0.086534731 & 0.168944387 & 0.044107563 \\
\hline Triangular & 0.248191625 & 0.240079842 & 0.019563879 \\
\hline Epanechnikov & 0.271915343 & 0.321858436 & 0.015571478 \\
\hline Quartic & 0.255419517 & 0.076459075 & 0.033375721 \\
\hline Triweight & 0.330617447 & 0.188661291 & 0.055776335 \\
\hline Tricube & 0.295160775 & 0.04851035 & 0.041241646 \\
\hline Gaussian & 0.128209034 & 0.202579582 & 0.025291028 \\
\hline Cosine & 0.261047996 & 0.295535463 & 0.017971944 \\
\hline Logistic & 0.158502473 & 0.193299944 & 0.033554477 \\
\hline
\end{tabular}

We compute again the IV and SPD surface using unconstraint semiparametric estimation proposed in [2], but now with a non fixed calendar bandwidth. In Figure 5 the results are computed using the following calendar bandwidth: $h_{\tau}=0.4$ for $\tau \leq 0.5, h_{\tau}=0.6$ for $0.5<\tau<1$ and $h_{\tau}=1$ for $\tau \geq 1$.

The computations are done again with Epanechnikov kernel function and with three representative moneyness bandwidths $h_{\kappa}=0.03,0.04,0.06$. If we compare these results with those we obtained for fixed calendar bandwidth we can notice that the arbitrage measure seems to smaller for all choices of moneyness bandwidth. This behavior is due to the fact that the main arbitrage occurs for long maturities so a smaller bandwidth doesn't include those maturities in the estimations. On the other hand, with a large fix calendar bandwidth the estimation for the shorter maturities are in some 
way disturbed by the turbulence that persist for the long maturities.

Finally we compute also the Calendar arbitrage measure as the volume of negative first derivative of total variance, see [2]. In this case we do not observe any violation of Calendar arbitrage free condition. We demonstrate it in Figure 4 where we show that indeed the total variance is strictly increasing in the calendar (maturity) direction for all moneyness values.

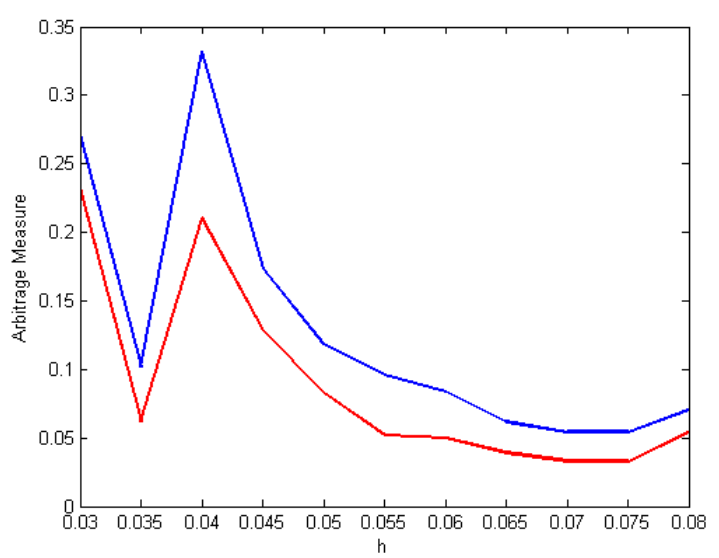

Figure 4. Arbitrage Measure for fixed calendar bandwidth (blue line) versus Arbitrage Measure for increasing calendar bandwidth (red line)

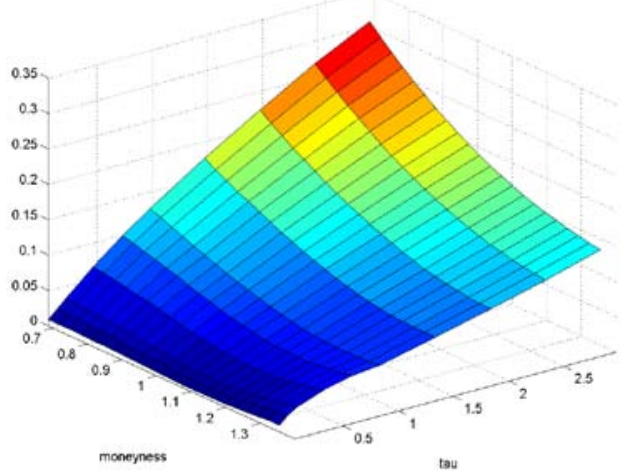

Figure 5. Total variance

\section{CONCLUSION}

In many cases, there is no way to valuate an option but to use implied volatility extracted from market prices. Since the moneyness and maturity of implied volatilities often do not match the data of valuated options, some sort of smoothing and interpolation is necessary. However, it can lead to arbitrage opportunity, if no-arbitrage conditions (nonnegativity of SPD) are ignored. In this paper, we analyzed the behavior of SPD (state price density) with respect to changes in bandwidth parameter. Using option data on DAX index it was documented that the no-arbitrage violating intervals of moneyness as well as the total area of SPD under zero heavily depends on the choice of this parameter. We observed that as the bandwidth parameter increases the degree of no-arbitrage violation decreases. Moreover, for $h>0.053$ the no-arbitrage conditions were satisfied, because the intervals of violations disappeared.

\section{ACKNOWLEDGMENT}

The research was supported by the Czech Science Foundation under project 13-25911S. The second author was supported also through the European Regional Development Fund in the IT4Innovations Centre of Excellence project $(C Z .1 .05 / 1.1 .00 / 02.0070)$ and the European Social Fund (CZ.1.07/2.3.00/20.0296) and SP2015/15, an SGS research project of VSB-TU Ostrava. The support is greatly acknowledged. All computations were done in MatLab 2009 and GAMS 23.5.

\section{REFERENCES}

[1] Bakshi, G., Cao, C., Chen, Z. Empirical performance of alternative option pricing models.Journal of Finance 52, 2003-2049, 1997.

[2] Benko, M., Fengler, M., Hardle, W., Kopa, M. On Extracting Information Implied in Options. Computational statistics, vol. 22, pp. 543-553, 2007.

[3] Black, F., Scholes, M. The pricing of options and corporate liabilities. Journal of Political Economy, vol. 81, p. 637-659, 1973.

[4] Dupire, B. Pricing with a smile. Risk Magazine 7 (1): 18-20, 1994.

[5] Glover, J., Ali, M.M. Using radial basis functions to construct local volatility surfaces. Applied Mathematics and Computation 217, pp. 4834-4839, 2011

[6] Rubinstein, M. Implied binomial trees. Journal of Finance 69: 771-818, 1994.

[7] Tichý, T. Lévy Processes in Finance: Selected applications with theoretical background. SAEI, vol. 9. Ostrava: VŠB-TU Ostrava, 2011.

[8] Yan, S. Jump risk, stock returns, and slope of implied volatility smile. Journal of Financial Economics 99: 216-233, 2011. 